\title{
GIF++: The new CERN Irradiation Facility to Test Large-Area Detectors for the HL-LHC Program
}

\author{
Roberto Guida ${ }^{1}$ \\ CERN \\ Geneva, Switzerland \\ E-mail: Roberto. Guida@cern.ch
}

on behalf of the EN, EP and AIDA GIF++ collaboration

\begin{abstract}
The high-luminosity LHC (HL-LHC) upgrade is setting a new challenge for particle detector technologies. The increase in luminosity will produce a higher particle background with respect to present conditions. To study performance and stability of detectors at LHC and future HL-LHC upgrades, a new dedicated facility has been built at CERN: the new Gamma Irradiation Facility $(\mathrm{GIF}++)$. The GIF++ is a unique place where high energy charged particle beams (mainly muons) are combined with gammas from a $14 \mathrm{TBq}{ }^{137}$ Cesium source which simulates the background radiation expected at the $\mathrm{LHC}$ experiments. Several centralized services and infrastructures are made available to the LHC detector community to facilitate the different R\&D programs.
\end{abstract}

38th International Conference on High Energy Physics

3-10 August 2016

Chicago, USA

\section{${ }^{1}$ Speaker}




\section{Introduction}

The current LHC configuration is set up to produce proton-proton collisions at a center-ofmass energy of $14 \mathrm{TeV}$ and a luminosity of $10^{34} \mathrm{~cm}^{-2} \mathrm{~s}^{-1}$. The high luminosity LHC (HL-LHC) project aims for an almost tenfold increase in luminosity [1]. Therefore, at the HL-LHC, the experiments will have to sustain rates about 10 times higher than in the present LHC operation period. The muon detectors situation is particularly critical in the forward regions where simulations on particle generation and absorption predict rates of the level of several $\mathrm{kHz} / \mathrm{cm}^{2}$.

A detailed knowledge of the detectors performance in such new hostile environment is therefore needed for the validation of ongoing upgrade programs. Two mechanisms are known affecting the detector response in presence of background: the detector occupancy and the ageing. The first is related to the specific detector efficiency to the background radiation, while the latter to the integrated particle fluence, dose and current in the detector. This demands a new series of studies on detector performance and stability, which were not possible at the old GIF facility. The old GIF facility was created in the SPS West Area in the mid 90ies [2]. All the muon detectors currently operational at the LHC experiments were validated at the old GIF. The old GIF combined gamma irradiation from a ${ }^{137} \mathrm{Cs}$ source with high energy charged particle beams. However, considering the background radiation increase expected at HL-LHC, the old GIF was not anymore suitable for the validation of the coming detector upgrade programs. To provide continuity and solutions to the new physics requirements, the new Gamma-Irradiation Facility (GIF++) is equipped with a stronger ${ }^{137} \mathrm{Cs}$ source and it is installed on the H4-SPS secondary beam line [3]. The GIF++ started operation in April 2015. Figure 1 shows a picture of the new irradiation facility.

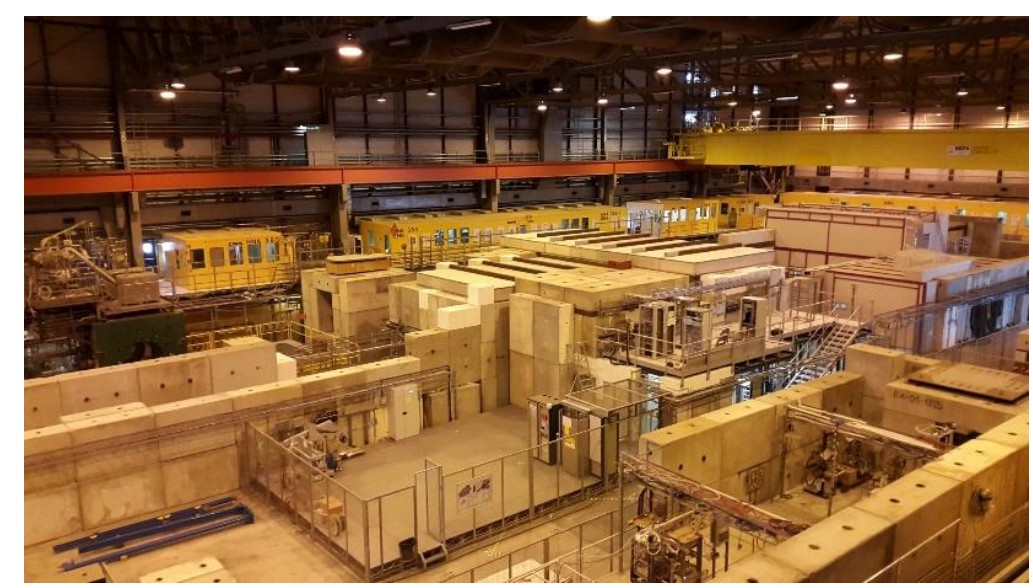

Figure 1 View from outside of the new GIF++ facility. The irradiation bunker as well as the preparation area and the electronic and service areas are visible. The total length of the facility is about 30 meters

The GIF++ is a joint project between the CERN Engineering (EN) and the Experimental Physics (EP) Departments. The EN Department provided the general infrastructure for housing irradiator, detectors and the beam line elements. The EP department is the main contact with the detectors users' community (definition of the requirements and daily coordination of the activities). Moreover, EP provided the irradiator, the infrastructure for the operation of gaseous 
detectors (i.e. gas systems). Several specific user infrastructures have been installed within the framework of the FP7 AIDA project [4].

\section{Overview of the GIF++ facility}

The GIF++ combines a $14 \mathrm{TBq}{ }^{137} \mathrm{Cs}$ source (about 30 times higher intensity compared to the old GIF), with high energy charged particle beams from the secondary SPS beam line H4 in CERN Prevessin EHN1 (887) building. The momentum of the beam line can range from $10 \mathrm{GeV} / \mathrm{c}$ up to the maximum SPS momentum of $450 \mathrm{GeV} / \mathrm{c}$. Secondary beams are produced directly from the impinging primary beam from SPS onto the T2 target. A spectrometer constructed of six MBN dipole magnets (total bending angle $42 \mathrm{mrad}$ ) is used for the momentum selection. The maximum momentum acceptance of the beam line is $\Delta \mathrm{p} / \mathrm{pmax}= \pm 1.4 \%$ [5]. Located near the end of $\mathrm{H} 4$, the GIF++ will be the main user of the beam line for 6-8 weeks per year. Gamma irradiation is available all year long, except during short maintenance periods (typically few days per year).

The GIF++ irradiation bunker has a total surface of $100 \mathrm{~m}^{2}$ divided in two regions (upstream and downstream the irradiator with respect to the beam line direction). The large surface available allows to test simultaneously several real size LHC-detectors (up to several square meters) as well as small prototype detectors. Figure 2 shows a drawing of the irradiation bunker where the main components and the surface available for the gamma irradiation are highlighted.

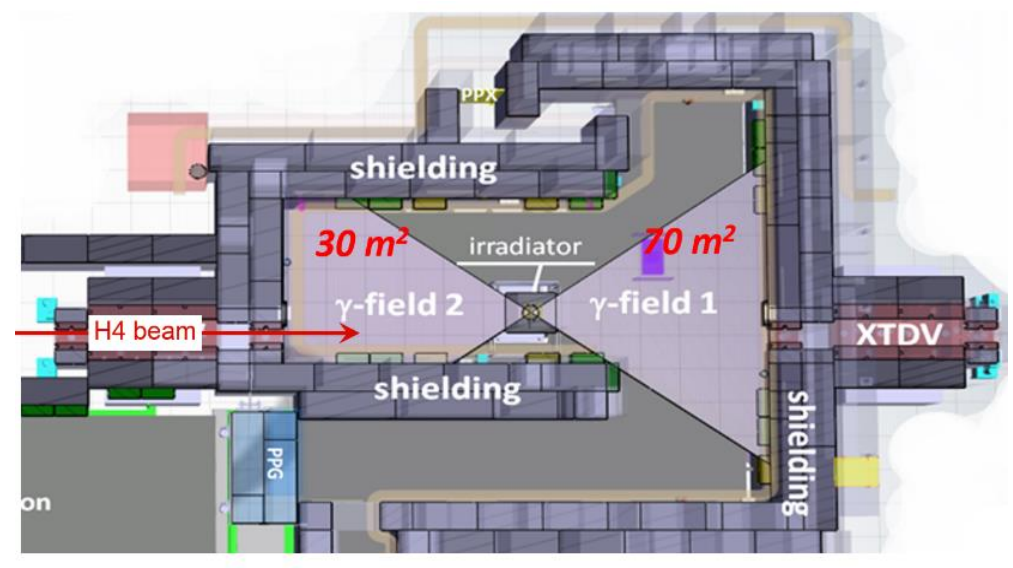

Figure 2 Inside view of the GIF++ irradiation bunker. The position of the irradiator and the surface of the two irradiation areas are indicated.

More than 20 setups are under test at the new facility. They include seven different gaseous detector technologies: Drift Tubes, Gas Electron Multiplier, Cathode Strip Chambers, MicroMegas, Resistive Plate Chambers, glass based Resistive Plate Chambers and Thin Gap Chambers. In addition to $\mathrm{R} \& \mathrm{D}$ and detector long term validation, two research groups are planning to perform quality acceptance tests of the final production chambers at GIF++ just before their installation on the LHC-experiments.

The irradiation area is a newly constructed bunker shielded by a $1.6 \mathrm{~m}$ thick concrete wall. It will be equipped with a material access door to allow easy installation of large detectors (currently the door is substituted by two removable concrete blocks). When needed, the roof of the bunker can be opened for the installation of very large detectors. Alongside the bunker a two- 
floor area has been prepared and it is hosting gas systems and electronic services. Each floor contains about 20 racks.

The facility is equipped with a raised floor allowing effective distribution of gas lines and cables. The shortest cable path from the electronic racks to a position in front of the irradiator is about 13 meters long.

A large preparation zone (about $80 \mathrm{~m}^{2}$ ) is available for preliminary tests before moving the detectors into the irradiation bunker. Irradiation bunker and preparation zone are equipped with gas lines, electricity and network.

For about 3 weeks per year a beam pipe traversing all the irradiation bunker need to be installed to allow operation of a downstream test area with electrons beam. During this period the irradiation from the Cs source can nevertheless continue (to free the space for beam pipe installation and dismantling some setups will be forced to temporarily move laterally).

The GIF++ is equipped with a powerful and flexible gas system infrastructure. All gases needed for the operation of particle detectors are available in the gas mixing zone through dedicated primary supply panels. Additional gas supply lines are available for R\&D program where testing of new gases is required. At regime, about 10 modules installed in the gas service area will be dedicated to the preparation of gas mixtures for particle detectors under test. The gas analysis system allows monitoring the flammability level of mixtures containing flammable components and measuring Oxygen and Water vapour contents on supply and return lines from detectors. The gas analysis module will perform automatic scans between all the gas streams selected. A gas chromatograph is permanently connected to the gas analysis rack allowing for more specific measurement of the gas quality. New gas recirculation systems have been designed for the GIF++ [6]. Two of this new module are currently successfully used by the CMS-CSC detector test and the R\&D program on gas systems upgrades.

From the gas mixing zone, 8 gas distribution panels for a total of 48 gas lines (each one with supply and return pipes) distribute the gas mixture to the irradiation bunker and the preparation zones.

The gas systems infrastructure is monitored using the same schema of the gas systems for the LHC experiments. All data about consumption, mixture composition, pressures ... are available through WinCCO interface and DIP protocol.

A Control System based on National Instruments CompactRIO WinCCO have been developer for the GIF++ facility. The purpose is to remote monitoring the facility as well as the attenuation filters of the irradiators and to archive all relevant parameters (irradiator source state, beam conditions, environmental conditions, access status, ...). The GIF++ Controls System redistributes interlocks (e.g. gas system faults) and all information about relevant parameters to user equipment.

An electronic logbook based on the web application package ELOG has been used to set-up a dedicated framework for operation and planning communications inside the large users' community [7].

\section{The ${ }^{137} \mathrm{Cs}$ irradiator and the gamma field}

The irradiator is a customized version of the GI-02 irradiator developed by the VF company. The ${ }^{137}$ Caesium source is embedded between two Tungsten blocks and can be moved from the 
garage position located at the bottom of the support tube to the irradiation position on the top of the tube (Figure 3$)$. The wide opening angle of both opening $\left( \pm 37^{\circ}\right)$ allows to irradiate a large fraction of the two irradiation zones. The irradiation window is located at beam height. Both openings are equipped with an Aluminum angular correction lens which makes the gamma flux uniform on a flat surface allowing to study the performance of large area particle detectors. For each irradiation zone, the gamma flux can be modulated independently (from 1 to 1/50000) using a system of six attenuation lens (the first made of Aluminum and the others of Lead) mounted on three remote movable supports (Figure 3). This system allows measuring the detector rate capability by comparing the detector efficiency in presence of different background radiation levels.
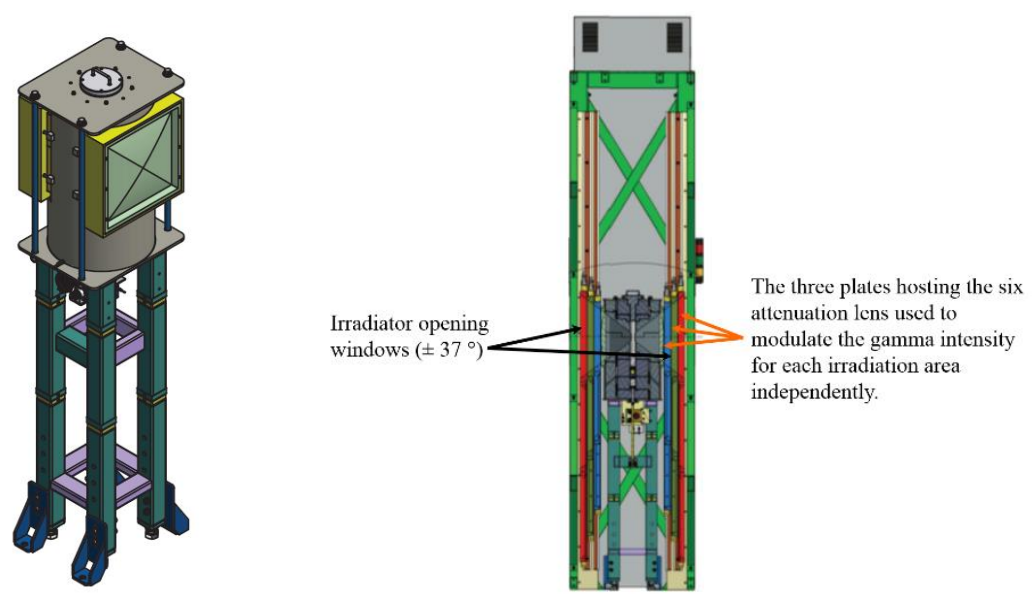

Figure 3 On the left, a view of the inside structure of the ${ }^{137}$ Cs GIF++ irradiator. On the right, a technical drawing of the GIF++ irradiator complemented with the system designed for an independent gamma flux modulation for the two irradiation areas.

The radiation field has been characterized during an extensive measurement campaign [8][9]. The dose has been measured in several positions to verify and tune the simulation. Figure 4 shows the gamma field in a condition when both sides are fully open to radiation (no attenuation applied). The dose rate at about $0.9 \mathrm{~m}$ from the $137 \mathrm{Cs}$ source is $470 \mathrm{mSv} / \mathrm{h}$. The GIF++ 137Cs source has been selected to optimize the operation of the facility for the validation of muon gaseous detectors. In fact, detectors under test at GIF++ can reach the expected HL-LHC accumulated dose in a reasonable period (Table 1), i.e. long enough to be representative of real detector operation but also allowing to achieve on-time the expected R\&D objectives. Other detector technologies requiring higher dose levels (for example Silicon detectors and calorimeters) cannot be efficiently tested at GIF++. However, other CERN irradiation facilities (IRRAD and CHARM) can be suitable for such tests.

Figure 5 shows a simulation of the bunker when only the downstream collimator is open. The presence of scattered radiation on the upstream side is visible. However, the radiation is almost a factor 10000 lower with respect to the downstream zone indicating that the two areas can be considered independent with good approximation. 
Table 1 Dose rate expected at the GIF++ facility compared to the maximum dose integrated by particle detectors at the future HL-LHC experiments. The GIF++ was intentionally designed to fulfil the requirements for testing large area muon detectors. Other CERN facilities are available for Silicon detectors.

\begin{tabular}{|c|c|}
\hline $\begin{array}{c}\text { Max. expected doses } \\
\text { at HL-LHC }\end{array}$ & $\begin{array}{c}\text { Equivalent time at GIF++ } \\
(\sim 1 \mathrm{~m} \text { from source })\end{array}$ \\
\hline Si-trackers: $\sim$ MGy/y & $>>$ years Other CERN \\
\hline Calorimeters: $\sim$ tens kGy/y & $<1$ year $\quad$ facilities available \\
\hline Muon systems: $\sim<\mathrm{Gy} / \mathrm{y}$ & "days to months" $\rightarrow \mathrm{GIF}++$ \\
\hline
\end{tabular}

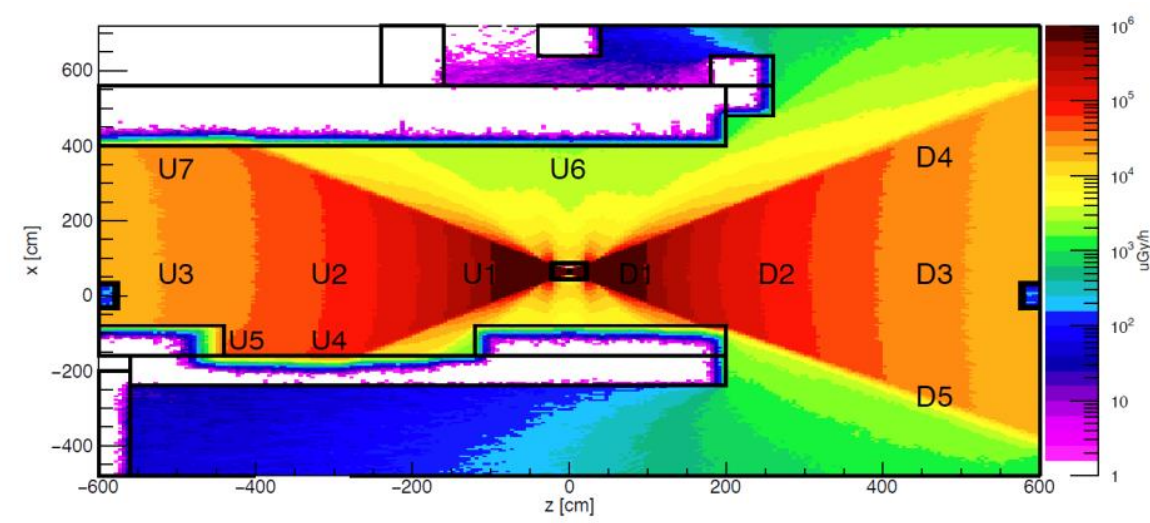

Figure 4 Gamma field simulation for the inside of the irradiation bunker when both sides are open without any attenuation lens [9].

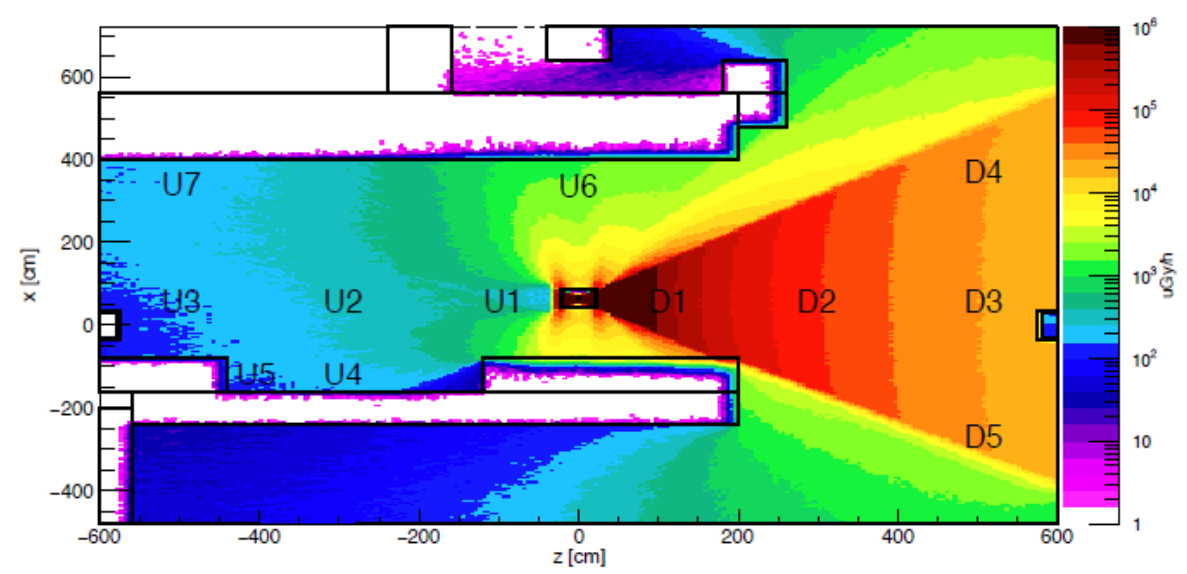

Figure 5 Gamma field simulation for the inside of the irradiation bunker when the maximum attenuation factor is applied for the upstream irradiation field and no factor is applied to the downstream area. A good independence of the two zones is visible (within almost a factor 10000) [9]. 


\section{The AIDA users' infrastructures}

Several user's infrastructures, provided within the framework of the FP7 AIDA project, are complementing the services for detector R\&D tests at GIF++. In particular, the AIDA project for GIF++ [10] includes the following detector specific infrastructures.

- Beam trigger

It is based on two TGCs chambers. At the moment, the TGC beam trigger is not operational due to some provision problems of the new front-end read-out electronics. It is temporary replaced with two plastic scintillators that in future will in any case complement the TGCs information.

- Cosmic trigger

The cosmic trigger is intended to provide a cosmic muon trigger when the beam trigger is not available (basically from November to April when the CERN accelerator complex is not operational). It will allow to monitor without interruption the detector efficiency. The Cosmic trigger is based on RPC detectors. It covers the downstream irradiation area and it is composed of three stations (one under the floor, the second on the floor and the third attached to the ceiling of the bunker). The installation of the detectors under the floor started in November 2015.

- Environmental and gas sensors

Several units equipped with temperature, humidity and pressure sensors are available inside the bunker and in the preparation area. They can be used to continuously monitor the gas parameter during $R \& D$ tests.

- Radiation monitors

Eight radiation sensors based on RADMON devices [11] are available inside the bunker area. They are attached to distribution manifolds located on the wall in two points. The sensors can be directly put in front of detectors under tests allowing to continuously monitor the accumulated dose all along the test independently from any other detector that could be installed at any time between the irradiator and a given detector during test periods.

\section{- Detector DCS}

The detector DCS monitors and controls all parameters $(\mathrm{HV}, \mathrm{LV}, \ldots)$ related to the beam and cosmic triggers. It is equipped with several other channels available for small users' setups to facilitate installation and test.

- Centralized DAQ

A centralized DAQ system is under development to acquire and distribute the information from beam and cosmic trigger to all detectors under test connected to the system.

\section{Conclusions}

The new CERN-GIF++ Gamma Irradiation Facility started operation in April 2015. The $\mathrm{GIF++}$ is a unique place for $\mathrm{R} \& \mathrm{D}$ tests where are simultaneously available a strong gamma source, particle beam, excellent gas and electronic infrastructures, unified control/monitoring system, setups for beam-cosmic trigger, radiation monitoring, environmental monitoring, DAQ, ...

Since the first day of operation, the facility is intensively exploited by a large collaboration from all LHC experiments (Figure 6). The efficiency of the facility in providing good conditions for detectors R\&D is greater $80 \%$. The installation of the HVAC system and other beam line elements during 2016 have further improve the stability of test conditions. 
The GIF++ community groups together more than 100 physicists, engineers and technicians from all the CERN LHC-experiments. Several different real-size detectors from all experiments are taking data. Seven different gaseous detector technologies are under validation for the future HL-LHC upgrade programs.

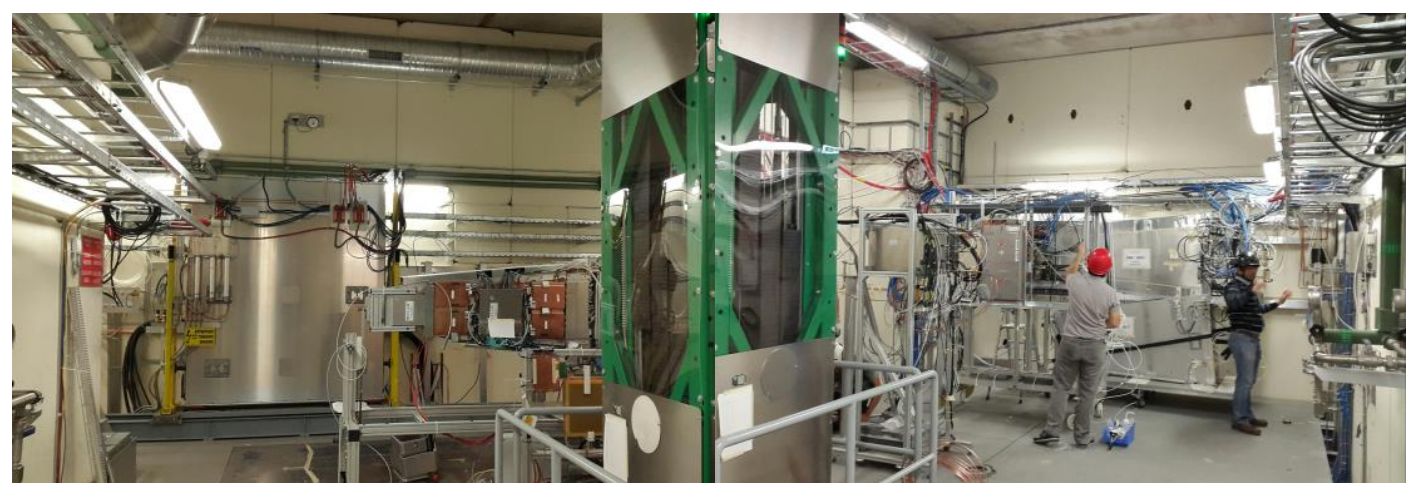

Figure 6 Inside view of the upstream irradiation field. Several detectors are visible. The ${ }^{137}$ Cs irradiator is also visible in the foreground.

\section{References}

[1] L. Evans and P. Bryant (editors), LHC Machine, JINST 3 (2008) S08001.

[2] S. Agosteo et al. A facility for the test of large area muon chambers at high rate NIMA 452 (94-104) 2000.

[3] M. Capeans, R. Fortin, L. Linssen, M. Moll, C. Rembser; A GIF++ Gamma Irradiation Facility at the SPS H4 Beam Line, CERN-SPSC-2009-029 / SPSC-P-339, 2009.

[4] R. Guida; GIF++: The New CERN Irradiation Facility to Test Large Area Detectors for the HL-LHC Program. CERN-PH Detector Seminar (https://indico.cern.ch/event/387753/).

[5] CERN Secondary Beam and Areas, http://cern.ch/sba.

[6] B. Mandelli et al., A new portable gas recirculation unit for gaseous detector R\&D, CERN EDMS note 1734199 .

[7] B. Mandelli et al., The GIF++ electronic logbook, CERN EDMS note 1734175.

[8] B. Biskup et al., Radiation-protection shielding of the gamma irradiation facility in EHN1, CERN EDMS note 1326312.

[9] D. Pfeiffer et al., The Radiation Field in the New Gamma Irradiation Facility at CERN, http://arxiv.org/abs/1611.00299.

[10] AIDA Milestone Report MS29: GIF++ User Infrastructure, https://cds.cern.ch/record/1497198/files/AIDA-MS29.pdf.

[11] F. Ravotti et al., Radiation Monitoring in Mixed Environments at CERN: from the IRRAD6 Facility to the LHC. Experiments, IEEE Trans. Nucl. Sci, vol. 54 (4), pp. 1170-1177, 2007. 